\title{
FROM DISMISSAL TO TRIUMPH IN SCIENTIFIC PUBLICATION
}

Khushbu Adhikari, ${ }^{1}$ Anand Kumar ${ }^{2}$

\begin{abstract}
For many aspiring researchers publishing their work in high-impact journals is a dream. An article is a publication that illustrates one or more outcomes of a well-designed and executed research. Scientific publishing is an essential aspect of medical progress where new advances in human knowledge are communicated to the outside world through it. Therefore, it is crucial that this knowledge is accurate, valid, reproducible, and of clinical use. One should follow the protocol provided by the journals for their dreams to become reality while writing research articles. In this paper, we discuss the points to focus in order to avoid rejection and increase the likelihood of getting published by providing what editors look for in a successful manuscript.
\end{abstract}

KEYWORDS Journal, Publication, Quality, Rejection, Research.

1. Department of Periodontology and Oral Implantology, Universal College of Medical Sciences, Bhairahawa, Nepal

2. Department of General Surgery, Universal College of Medical Sciences, Bhairahawa, Nepal

DOI: http//doi.org/10.3126/jucms.v7i2.27145

For Correspondence

Dr. Khushbu Adhikari

Department of Periodontology and Oral Implantology

Universal College of Medical Sciences,

Bhairahawa, Nepal

E-mail:adhkhush@gmail.com 


\section{INTRODUCTION}

Journal rejection rates can vary widely in scientific publication. The common reasons cited for rejection of publication has been listed in various studies. ${ }^{1-3}$ Rejection can happen to authors who are early, as well as those established in their careers. For most authors, rejection is difficult to deal with, yet many have faced this unpleasant prospect at some point in their career. This can be very discouraging to a researcher who spend years planning and executing their research and making conclusions that could benefit the larger community. It is always recommended to have a sound knowledge about format of the journal and know its rejection rates beforehand. Knowing the most common reasons for rejection will help ensure that the manuscript receives a thorough review so that one can address any concerns and resubmit accordingly. Many papers originally rejected may ultimately find a more suitable journal if correction of errors that lead to rejection is emphasized. Any error related to study design cannot be corrected once the study is complete, but many others can be rectified at the manuscript writing stage. Therefore, certain steps should be considered before submitting a manuscript to journal for publication.

The errors subjecting a manuscript for rejection of publication can be broadly discussed in various categories:

a. Initial screening and reviewing of manuscript.

b. Issues related to quality and presentation of research.

c. Issues related to the journal interest.

d. Other issues.

\section{A. Initial screening and reviewing:}

Initially, an article is screened for any error in the technical aspect before it is send for review or to the editor-in-chief for consideration. The major issues considered at this stage are:

1. The contents of the article if it is plagiarized, or is currently under consideration or review at another journal.

2. The article does not conform to the guidelines for authors of the journal.

3. References are either incomplete or irrelevant and not presented in an updated and specified style.

4. It does not meet the aim and scope of the journal. The article does not focus on the specificity of journal. Hence, it does not fulfill the aim and scope of the journal.

5. An incomplete manuscript, which may be lacking key elements such as the title, authors, affiliations, keywords, main text, references and all tables and figures. The figures are incomplete or unclear. The article does not depict a complete study but contain observations. It discusses few findings of the work in the field ignoring other important aspects.

6. The defects in methodology and data analysis is evident. The study lacked clear control groups or other comparison parameters. The study did not conform to recognized procedures or reproducible methodology. The analysis is not statistically valid.

7. The conclusion is unjustified based on rest of the article. The discussion is illogical, unstructured or invalid. The data does not support the conclusions. The conclusions ignore large portion of the literature.

8. When the work seems an extension of a different article from the same authors: The work is clearly extraction of a larger study to make as many articles as possible mostly from the same authors.

9. The language, structure, or figures to be assessed are not up to the mark. The use of English is not sufficient and clear enough for the peer review process.

10. It is not factual and is of marginal interest to the field. The question behind the work is of no interest to the readers of the specific journals.

\section{B. Issues related to quality and presentation of research}

Even a well-written article will not cover errors in study design. Therefore, this fundamental problem must be resolved in the initial stages of conceptualizing the study. The best way to prevent such errors is to do a thorough review of literature and determining the best methodologies for conducting own research.

\section{Study design}

1. The errors in study design includes poorly formulated research question and inadequacy of approach to answering the research question.

2. The errors in calculating appropriate sample and not following reliable method that is suitable for the problem to be studied.

3. The errors concerning unreliable or incomplete data and use of inappropriate statistical analysis tools.

\section{Writing and organization}

It is very important for authors to present a rational argument 
in their papers. It should be convincing to readers that the research is important and create interest through writing.

The reader or reviewer may lose interest in the article if there is:

1. An incomplete introduction that does not establish the background of the problem being studied.

2. Rationale for the study is not explained sufficiently.

3. Literature review is not thoroughly described and updated.

4. Discussion that only repeats the results without proper interpretation.

5. The study data does not support the conclusions.

6. Manuscript is unable to place the study in a broad context.

\section{Inadequate preparation of the manuscript}

Few authors may face additional problem because of their style of writing, as peer reviewers do not always distinguish between the manuscript content and style of writing. Thus, such manuscripts may get negative comments even if the research is of high quality.

However, these problems are easily fixable, either by asking a colleague to review the paper or by getting professional help in editing and formatting the paper.

1. Failure to follow the journal's instructions for authors and the title, abstract, and/or proper formatting of the cover letter.

2. Errors related to grammar and spelling along with unclear sentences.

3. Errors in designing concise tables or figures.

\section{Issues related to the journal's interest:}

It's very important to know the aim and scope of the specific journal for which the article is being prepared for. Journals can be a collection of an ongoing conversation about a particular topic. Therefore, it is important to always see whether papers with similar topic have been published in the past in the journal you are aiming for. If no similar work has been published prior, there may well be a reason, namely, the journal isn't interested in the topic. Many manuscripts are rejected instantly by journals before the peer review, because the manuscript does not fit into the aim and scope of the journal appropriate for the journal's audience. ${ }^{5,6}$

1. Findings that are relevant to a small audience and journal is not specific about it.

2. Manuscripts not following the format specified by the journal.

3. Manuscripts that do not fall under the aims and scope of the journal.

4. Topics that are repetition or of no interest to the journal.

\section{Other issues:}

Many authors tend to present their work as "this has never been studied before" to explain the significance of their paper. But this is not good enough for the study to be placed in a broader context. The reason of significance of their work should be specific, presenting it as a particular medical intervention which could affect a specific policy discussion, or change a conventional theory or belief.

1. The content of the article may not be timely.

2. The journal may not have space for the article.

3. The article cannot compete with other high quality papers of the journal.

4. The journal may have recently published another article on the subject.

\section{Publication bias.}

\section{Steps to increase the likelihood of publication:}

There are many articles which have been published guiding the author through the process of writing a manuscript in a stepwise fashion from start to finish. ${ }^{7-9}$ The authors emphasize on certain points for increasing the likelihood of publication.

1. Journal selection: We recommend spending some time in choosing the accurate journals for submitting your paper. You can create a list of journals and review your options before deciding which journal to submit your manuscript to. It is also equally important to have a prior idea about the way other authors in the journal write their abstract, headings, introductions and conclusions because that's what is expected from the reviewers and readers to see.

2. Linguistic accuracy: The deficiencies in writing different sections of a manuscript can be due to the content being disorganized, incomplete and with no clear meaning. Hence, use of proper grammar and few typing errors can be easily considered by the editor. The use of simple language is suggested so that the results and discussion are to the point.

3. Consistent data: The data presented should be consistent throughout the article or it gives impression of a negligent work to the reviewer. The data presented in tables should match which those presented in the text. Hence allow yourself 
enough time to re-evaluate the legends and number used in tables and that mentioned in the text.

4. Thorough review by colleagues: It is always wiser to ask one or few colleagues for a manuscript review prior to sending it for publication. As, going through our own manuscript several times, we tend to overlook our mistakes. Additionally, our colleagues can provide with new insight on the paper with different opinions and help predict the reviewer's reaction to the manuscript.

5. Data presentation in logical manner: While writing the manuscript, one should ensure proper flow of information through simultaneous data presentation. The authors should also be careful about the tables and figures to include for orderly and logical presentation of data for the readers to have same conclusions. Anticipating critics from the readers and modifying or adding data necessary to avoid them is another way to go by for a successful publication.

\section{Summary}

The process of publishing a manuscript in a high-impact journal is possible and starts with choosing an important research question and designing a sound study with use of appropriate statistical tool. The research work should be performed with impeccable integrity and attention to detail. The next steps include writing the manuscript, submitting it to the right journal, responding to reviewer comments positively, and completing the standard procedure. It is more than satisfying to see hard work pay off while publishing a highquality article which adds and updates the current knowledge

\section{REFERENCES}

1. Smith MU, Wandersee JH, Cummins CL. What's wrong with this manuscript? : An analysis of the reasons for rejection given by Journal of Research in Science Teaching reviewers. Journal of Research in Science Teaching. 2003; 30(2): 209-211.

2. Pierson DJ. The top 10 reasons why manuscripts are not accepted for publication. Respiratory Care. 2004; 49(10): 124652.

3. Byrne DW. Common reasons for rejecting manuscripts at medical journals: A survey of editors and peer reviewers. Science Editor. 2000; 23(2): 39-44.

4. Zimmer C. It's science, but not necessarily right. The New York Times. 2011; June 25.

5. Ajao OG. Some reasons for manuscript rejection by peerreviewed journals. Annals of Ibadan Postgraduate Medicine. 2005;3(2):9-12.
6. Kumar M. A review of the review process: manuscript peerreview in biomedical research. Biology and Medicine. 2009; 1 (4): 1-16.

7. Fried PW, Wechsler AS. How to get your paper published. J Thorac Cardiovasc Surg. 2001; 121[4 suppl]:S3S7.

8. Kern MJ, Bonneau HN. Approach to manuscript preparation and submission: how to get your paper accepted. Catheter Cardiovasc Interv. 2003; 58:391-6.

9. Kliewer MA. Writing it up: a step-by-step guide to publication for beginning investigators. Am J Roentgenol. 2005;185: 591-6. 